\title{
Evaluación de un programa escolar en Costa Rica basado en habilidades para vivir
}

\author{
Julio Bejarano; Federico Ugalde; Desirée Morales
}

Instituto sobre Alcoholismo y Farmacodependencia, Costa Rica.

Enviar correspondencia a:

Julio Bejarano. Apartado 4494-1000, San José, Costa Rica. Correo-e: juliobejarano@costarricense.cr

\section{RESUMEN}

La necesidad de evaluar un programa de prevención desarrollado bajo el paradigma de las habilidades para vivir, llevó a diseñar una estrategia investigativa mediante la cual se analizó información acerca del desempeño de una muestra de adolescentes en una serie de dominios (toma de decisiones, relaciones interpersonales, comunicación asertiva, percepción de amenazas, autoestima, presión de grupo) y sobre consumo de drogas, antes y después de la aplicación del programa denominado Trazando el Camino a una población estudiantil costarricense en el año 2001.

Para el logro del objetivo propuesto se trabajó con un diseño pretest-postest, dentro de una modalidad de investigación cuasi-experimental. Se aplicó un instrumento de medición a una muestra de 1879 en el pretest y a otra de 920 jóvenes estudiantes en el postest.

Los resultados obtenidos son de interés, en la medida en que tanto en el grupo al cual se le aplicó el programa, como aquel que operó como grupo de comparación, se registraron mejoras significativas en la autoestima, manejo del estrés, presión grupal y comunicación. Esto puede interpretarse en función de la evolución propia de los adolescentes en este período. También es probable que los jóvenes, en ambos grupos, estuviesen expuestos a influencias preventivas adicionales, que escaparon al conocimiento y manejo de los investigadores. Se considera que el período de evaluación del programa debería ser mayor, con el propósito de conocer sus posibles efectos en el largo plazo, situación de importancia en la medida que los programas mismos tengan permanencia en el tiempo.

Palabras clave: estudiantes, consumo drogas, Costa Rica, habilidades para vivir, programa prevención escolar, evaluación.

\section{ABSTRACT}

The need to evaluate a prevention programme developed under the paradigm of life skills, led to the design of an investigative strategy by means of which information was analysed on the performance of a sample of adolescents in a number of life skills (decision making, face-to-face relationships, communication, stress handling, self-esteem, peer pressure) and on drug use, before and after the application of the Trazando el Camino Programme on a Costa Rican student population in 2001.

In order to achieve the proposed objective, a pretest-posttest design was used, within a quasi-experimental framework. A sample of 1897 young students completed a questionnaire in the pretest and 920 in the posttest.

The results were interesting insofar as both the group where the programme was applied as well as the control group showed significant improvements in self-esteem, stress management, peer pressure and communication. This could be interpreted in respect of the typical evolution of adolescents during this period. It is also probable that the young people in both groups were exposed to additional preventive influences which were outside the knowledge and control of the researchers. It is considered that the evaluation period of the programme should be longer, with the aim of ascertaining its possible long-term effects, an objective of some importance as this programme is being implemented over the long term.

Key words: students, drug use, Costa Rica, life skills training, school prevention program, evaluation.

\section{INTRODUCCIÓN}

$\mathbf{S}$ in lugar a dudas el consumo de tabaco, alcohol y otras drogas es una situación que afecta a la juventud costarricense y que requiere una estrategia nacional para desestimular el consumo en quienes se han iniciado y ofrecer una alternativa de prevención para evitar que quienes no han tenido contacto con estas drogas lo posterguen o no lo hagan.

En estudios realizados en Costa Rica por el Instituto sobre Alcoholismo y Farmacodependencia (I.A.F.A) en la población adolescente de 12 a 24 años (Bejarano 
y San Lee, 1997), se encontró que en el último año $35,2 \%$ de los jóvenes habían tomado alcohol, 13,3\% habían fumado, 1,3\% refirieron consumo de benzodiacepinas sin prescripción y 0,5\% consumo de drogas ilícitas. En el sector educativo, los y las jóvenes con edades entre 15 y 17 años muestran niveles de prevalencia superiores, al ser el consumo del último mes igual a 33,2\% para alcohol, 21,5\% para tabaco, 3,3\% para marihuana y $0,9 \%$ para cocaína y para "crack" (Ugalde et.al. 2003). La experiencia de evaluar constructos como rebeldía y depresión reveló que los consumidores de tabaco y alcohol presentaban dos veces más riesgo de mostrar síntomas de depresión y rebeldía en tanto que en los consumidores de drogas ilícitas el riesgo era cuatro veces mayor (Ugalde et.al. 2003).

Por ser la problemática del consumo de drogas y la adolescencia, aspectos prioritarios del programa de Gobierno se elaboró una respuesta preventiva y se formularon directrices desde el Ministerio de Salud y el Viceministerio de la Presidencia (Mideplan, 2001; Ministerio de Salud, 2001; ICD, 2003), luego de establecerse que los programas de Habilidades para la Vida, eran los que demostraban mejores resultados a corto y largo plazos (Gorman, 1996, Becoña, 1999, Botvin, 1995). En cuanto a detección e intervención tempranas se estableció la importancia de capacitar al personal docente en habilidades para detectar tempranamente a los adolescentes afectados, con el fin de que realicen una intervención específica.

En el año 1999 se presentó el programa de Habilidades para vivir, denominado "Trazando el Camino", que surgió como una propuesta educativa orientada hacia los y las jóvenes de tercer ciclo de educación general básica, con el fin de prevenir el consumo y el abuso de drogas.

Este proyecto se llevó a cabo bajo el patrocinio técnico y financiero inicial de la Organización Panamericana de la Salud (OPS), y se desarrolló bajo el liderazgo del Instituto de Alcoholismo y Farmacodependencia (I.A.F.A), en coordinación interinstitucional con el Ministerio de Educación Pública y el Centro Nacional de Prevención Contra las Drogas (CENADRO), hoy Instituto Costarricense Contra las Drogas (I.C.D.).

El programa pretende hacer transformaciones en los conocimientos de los y las jóvenes y los capacita para desarrollar competencias en aspectos fundamentales de su desarrollo y para resistir el consumo de drogas. Contempla habilidades para mejorar la autoestima, la asertividad, la comunicación, el manejo de la ansiedad, la toma de decisiones, las relaciones familiares, el manejo de la presión del grupo, la influencia social y la información básica sobre drogas.
El objetivo del programa es disminuir el consumo de drogas en la población estudiantil de todo el país al desestimularlo, evitar o retardar la edad de inicio y favorecer la cesación del consumo mediante la adquisición de habilidades para vivir. El fomento de las habilidades para vivir ha cobrado vigencia en especial en la década anterior cuando se planteó fuertemente que entre estudiantes de secundaria el rendimiento académico y la competencia social podrían incrementarse mediante el fomento de habilidades tales como: comunicación, relación entre pares, asertividad, resistencia al consumo de drogas y fortalecimiento del compromiso personal contra el consumo (Botvin, et.al. 1995; Scheier, et.al. 1999) La Organización Mundial de la Salud (OMS, 1993) menciona, complementariamente, el manejo de emociones, el autoconocimiento, el pensamiento creativo y la toma de decisiones.

Según sus gestores (Botvin, Baker, et.al., 1990), las evaluaciones de largo plazo desarrolladas a finales de la década de los ochenta revelaron que el entrenamiento en habilidades para la vida tuvo un impacto significativo sobre el fumado de tabaco y de marihuana y el consumo excesivo de alcohol, luego de tres años en los que los alumnos recibieron al menos un $60 \%$ del programa. Los hallazgos más importantes, sin embargo, se efectuaron luego de 6 años de aplicación del programa. Se encontró, por ejemplo, que el programa redujo en $40 \%$ el porcentaje de estudiantes que consumieron drogas en el último mes y en $66 \%$ la proporción de quienes consumieron en la última semana (Botvin, et.al., 1995).

Este trabajo constituye una evaluación del impacto de un programa de habilidades para vivir en adolescentes costarricenses escolarizados durante los años 2001-2002.

\section{MATERIALY MÉTODO}

Esta evaluación de un programa preventivo, aplicado a adolescentes que cursaron el $7^{\circ}, 8^{\circ}$, y $9^{\circ}$ años, se realizó mediante un diseño cuasi-experimental ${ }^{1}$ de pretest-postest en el 2001 y el 2002. Para ello se escogió una muestra nacional de colegios públicos diurnos, académicos y técnicos y se exploraron las habilidades para vivir, las características de consumo de drogas, el riesgo percibido de consumo, y las actitudes de los y las jóvenes hacia las sustancias psicoactivas.

El presente informe constituye un análisis comparativo de los resultados obtenidos tras la aplicación de un instrumento antes del desarrollo del programa (pretest) y después de finalizado el mismo (postest).

En este diseño, los sujetos no son asignados al azar a los grupos ni emparejados. Los conjuntos se forman con anterioridad a la realización del experimento. 
El primero tuvo lugar a inicios del curso lectivo y el segundo un año después.

Para la selección de la muestra se tomó en consideración el criterio de los funcionarios de las Sedes Regionales del I.A.F.A, los cuales ayudaron a determinar en cuales regiones se podría dar un buen seguimiento en la aplicación del proyecto. El seguimiento sería realizado por el IAFA y el Ministerio de Educación en los colegios en los cuales se aplicó el programa y en los colegios control. La muestra estuvo formada por 12 colegios de diferentes regiones que funcionaron como colegios experimentales y otros 12 que se constituyeron como grupo control. En los primeros se aplicaron las guías preventivas. En los colegios controles no.

En la muestra no se tomó en cuenta el tamaño del colegio ni la proporción de la población estudiantil en la región. Se partió del principio que en cada Región los colegios y las secciones por estudiar serían seleccionados aleatoriamente.

Se partió del hecho que, en promedio, en cada grupo había unos 30 estudiantes. Así, el tamaño estimado de la muestra fue de 2160 sujetos. Sin embargo, se redujo a 1879 en razón de que fue la población real al momento de aplicar el instrumento. Para la aplica-

\section{Tabla 1. Características sociodemográficas de la muestra total. Costa Rica, 2001-2002}

\begin{tabular}{|lcc|}
\hline VARIABLES & $\begin{array}{c}\text { PRETEST } \\
\text { (valores } \\
\text { porcent.) } \\
\mathbf{n = 1 8 7 9}\end{array}$ & $\begin{array}{c}\text { POSTEST } \\
\text { (valores } \\
\text { porcent.) } \\
\mathbf{n = 9 2 0}\end{array}$ \\
\hline Sexo & & \\
Masculino & 46.8 & 45.6 \\
Femenino & 52.4 & 53.0 \\
N.R & 0.8 & 1.4 \\
Edad & & \\
12 o menos & 11.3 & 0.3 \\
13-14 & 56.3 & 48.4 \\
15-16 & 28.7 & 45.8 \\
17 y más & 3.4 & 5.3 \\
N.R & 0.3 & 0.2 \\
Nivel Educativo & & \\
$7^{\circ}$ & 33.2 & -12.9 \\
$8^{\circ}$ & 32.9 & 54.3 \\
$9^{\circ}$ & 33.9 & 45.7 \\
Tipo de Colegio & & \\
Público-Académico & 80.9 & \\
Público Técnico & 18.5 & \\
NR & 0.6 & \\
\hline
\end{tabular}

ción del postest, el tamaño de la muestra varió, reduciéndose la misma a 920 estudiantes en virtud que el instrumento fue administrado solamente a los alumnos de octavo y noveno año. Escapó del control de los investigadores la posibilidad de aplicarlo en $10^{\circ}$ año a los alumnos que el año anterior cursaron $9^{\circ}$, en virtud de factores como el cambio de ciclo, el cual supone una muy alta deserción.

Los datos de la Tabla 1 indican que la edad promedio, así como la mediana, fue de 14 años (DE=1,57).

La recolección de la información se efectuó mediante un cuestionario compuesto por 126 reactivos, distribuidos en seis áreas por evaluar, conocidas como dominios. Estos dominios fueron: relaciones interpersonales, toma de decisiones, comunicación asertiva, autoestima, percepción de amenaza y presión grupal (véase Tabla 2). Además incluye preguntas sobre consumo de drogas y actitudes hacia ellas.

En el postest se administró el mismo instrumento, el cual fue desarrollado por la Oficina de Investigación del Instituto sobre Alcoholismo y Farmacodependencia. Su fundamento teórico reside en los postulados de la OMS acerca de las habilidades para vivir (OMS, 1993). La idea de construir el instrumento localmente obedeció a la ausencia de herramientas de esta naturaleza en el medio nacional que permitieran evaluar éste programa en específico, pese a que en el I.A.F.A se ha construido una apreciable cantidad de cuestionarios y se han validado y estandarizado algunos de factura internacional, pero no para efectos evaluativos.

Las preguntas de los seis dominios del la Tabla 2 están construidas como escalas tipo likert (Likert, 1976 y Papua, 1979) con cinco opciones (Siempre Casi siempre - A veces - Casi nunca - Nunca). El mayor puntaje obtenido en cada dominio es de cinco, porque las respuestas de los entrevistados son ponderadas por el total de preguntas en cada sección. Es posible obtener puntajes globales a pesar de la diversidad de las áreas evaluadas. Así, la ponderación de todos los dominios dio lugar a un puntaje global (como se detallan en la Tabla 8) cuyo máximo fue 5 puntos.

La sección de consumo de drogas se construyó a partir de la experiencia del I.A.F.A en esta materia. Consta de 25 preguntas que exploran edad de inicio, prevalencia de consumo de alcohol, tabaco, marihuana y cocaína y motivación del consumo. La sección de conocimientos cuenta con 9 reactivos en tanto que la de actitudes de 23.

El cuestionario incluye dos secciones adicionales. Una sobre conocimientos y actitudes hacia el alcohol (15 ítems), otra sobre conocimientos y actitudes hacia el fumado (17 ítems). 
Tabla 2. Dominios incluidos en el instrumento de evaluación de Programa Trazando el Camino, Costa Rica, 2001-2002

\begin{tabular}{|llcc|}
\hline DOMINIO & SIGNIFICADO & $\begin{array}{c}\text { NUMERO } \\
\text { DE PREGUNTAS }\end{array}$ & CONFIBIALIDAD \\
\hline $\begin{array}{l}\text { Toma de } \\
\text { Decisiones }\end{array}$ & $\begin{array}{l}\text { Mide la capacidad del joven para asumir } \\
\text { decisiones por sí solo. }\end{array}$ & 11 & 74,4 \\
$\begin{array}{l}\text { Relaciones } \\
\text { Interpersonales }\end{array}$ & $\begin{array}{l}\text { Mide la capacidad del joven para interactuar } \\
\text { socialmente. }\end{array}$ & 6 & 47,8 \\
$\begin{array}{l}\text { Comunicación } \\
\text { asertiva }\end{array}$ & $\begin{array}{l}\text { Mide la capacidad para comunicarse y } \\
\text { transmitir mensajes de manera efectiva }\end{array}$ & 12 & 40,7 \\
$\begin{array}{l}\text { Percepción de } \\
\text { amenazas }\end{array}$ & $\begin{array}{l}\text { Mide la forma como los jóvenes perciben } \\
\text { el entorno social }\end{array}$ & 8 & 42,5 \\
$\begin{array}{l}\text { Autoestima } \\
\text { Mide la opinión que los jóvenes tienen } \\
\text { de sí mismos }\end{array}$ & 6 & 17,6 \\
$\begin{array}{l}\text { Presión de } \\
\text { Grupo }\end{array}$ & $\begin{array}{l}\text { Mide la influencia que los grupos de pares } \\
\text { ejercen sobre los adolescentes que puede } \\
\text { interferir o no, en las decisiones }\end{array}$ & 7 & 31,3 \\
\hline
\end{tabular}

\section{RESULTADOS}

\section{Evaluación de los dominios}

Los resultados que se exponen el la Tabla 3, revelan cambios estadísticamente significativos en cuatro de los seis dominios estudiados. Este fenómeno ocurre tanto en el grupo control como en el experimental.

El dominio presión grupal constituye un área sensible, dado que fue el que experimentó la mayor transformación. Llama la atención que no haya diferencias entre los estudiantes que no llevaron el programa en comparación con los que sí lo recibieron. Una expli- cación tentativa estribaría en que los adolescentes más jóvenes no habrían hecho aún una ruptura con su grupo familiar como sucede a mayores edades, en las cuales la importancia de la influencia de los pares resulta relevante (Krausskopf, 1994). Esto podría explicar la mejoría observada en el postest. Cabe mencionar también la posibilidad de que los sujetos del grupo control estuvieren sometidos a alguna influencia preventiva, ajena al programa evaluado, la cual no se tuvo oportunidad de registrar y de dar seguimiento.

Un estudio reciente mencionó que "Los y las adolescentes mayores prefieren actividades directamente relacionadas con espacios de identificación

TABLA 3. Puntajes promedio obtenidos por los estudiantes de los grupos control y experimental en los dominios estudiados. Costa Rica, 2001-2002

\begin{tabular}{|lcccc|}
\hline DOMINIOS & $\begin{array}{c}\text { Pretest } \\
\text { CONTROLES }\end{array}$ & $\begin{array}{c}\text { Postest } \\
\text { CONTROLES }\end{array}$ & $\begin{array}{c}\text { Pretest } \\
\text { EXPERIM. }\end{array}$ & $\begin{array}{c}\text { Postest } \\
\text { EXPERIM. }\end{array}$ \\
\hline Toma de decisiones & 4.02 & $3.94\left(^{*}\right)$ & 3.99 & 4.01 \\
Relaciones Interpers. & 3.62 & 3.61 & 3.56 & $3.66\left(^{*}\right)$ \\
Comunicación Asertiva & 3.47 & $3.66\left(^{*}\right)$ & 3.41 & $3.65\left(*^{*}\right)$ \\
Autoestima & 3.69 & $4.13\left({ }^{*}\right)$ & 3.65 & $4.10\left(*^{*}\right)$ \\
Manejo del estrés & 2.83 & $3.79\left({ }^{*}\right)$ & 2.90 & $3.80\left(^{*}\right)$ \\
Presión grupal & 2.52 & $4.19\left(^{*}\right)$ & 2.53 & $4.21\left(^{*}\right)$ \\
\hline (*) Diferencia estadísticamente significativa entre el pretesty el postest. & & \\
\hline
\end{tabular}


con pares, lo que ha sido confirmado por diferentes postulados teóricos que sostienen que conforme se avanza en la fase adolescente los pares cobran un papel central y muchas veces determinante en la vida de las personas". (Valverde, et.al. 2001, pág. 55).

El dominio autoestima, tanto en uno como en otro grupo (experimental y control), revela una mejora significativa; que pareciera reflejarse en el desempeño de los jóvenes en los restantes dominios, especialmente en el de presión de grupo. De hecho, los mejores puntajes en el postest de los dos grupos ocurrió en este dominio, situación positiva si se toma en cuenta la necesidad del adolescente de saber que los otros piensan bien de él y, consecuentemente, que él mismo se sienta bien como persona. (Krauskopf, 1994; Papalia y Olds, 1990).

El dominio manejo del estrés y percepción de amenazas también mostró una mejora, pero sin alcanzar niveles óptimos (4 puntos o más). Idéntica situación se observa en la comunicación asertiva. Es posible que exista una relación de dependencia entre estos dominios, lo cual podría implicar que mejorías en unas habilidades redundarían en mejorías en otras. Nótese que prácticamente no existen diferencias entre la ejecución de los controles y aquellos en que se aplicó el programa.

El dominio toma de decisiones en los controles, disminuyó significativamente, mientras que en el grupo experimental se mantuvo. El de relaciones interpersonales no mostró cambios en el grupo control pero si en el experimental, aunque no alcanzó el nivel óptimo de 4 o más puntos.

Según se aprecia en la Tabla 4, y de acuerdo con lo que teóricamente se esperaría, no existen diferencias entre los valores obtenidos en los diferentes dominios, tanto en los grupos control como experimental.

Los dominios en los cuales ocurrieron aumentos significativos entre pre y postest, tanto para hombres como para mujeres fueron: autoestima, manejo del estrés y de la presión grupal. En comunicación asertiva sólo las mujeres muestran un aumento significativo, aunque éste no alcanza el nivel mínimo óptimo de 4 puntos. Proporcionalmente, las mujeres obtuvieron mayores incrementos entre el pre y el postest que los hombres.

Los dominios con mejor desempeño en hombres y en mujeres son los de autoestima y presión grupal. Lo que llama la atención es que esto haya ocurrido también en los grupos control, de donde se podría colegir, preliminarmente, que el programa, en un año plazo, no mostró los resultados que se esperaban; es decir, que se dieran cambios significativos en el grupo en el cual se aplicó el programa preventivo. Esta interpretación no necesariamente descalifica el programa, puesto que luego de su aplicación hubo mejoras en ellos. Falta por interpretar el resultado obtenido con los controles.

De acuerdo con estos resultados, las relaciones interpersonales y la comunicación asertiva son habilidades que las mujeres manejan de manera más adecuada.

Tabla 4. Puntajes promedio obtenidos por los estudiantes de los grupos control y experimental en los dominios estudiados, según sexo. Costa Rica, 2001-2002

\begin{tabular}{|c|c|c|c|c|c|c|c|c|}
\hline \multirow[t]{3}{*}{ DOMINIOS } & \multicolumn{4}{|c|}{ CONTROLES } & \multicolumn{4}{|c|}{ EXPERIMENTALES } \\
\hline & \multicolumn{2}{|c|}{ pretest } & \multicolumn{2}{|c|}{ postest } & \multicolumn{2}{|c|}{ pretest } & \multicolumn{2}{|c|}{ postest } \\
\hline & $\mathbf{M}$ & $F$ & M & $\mathbf{F}$ & $\mathbf{M}$ & $\mathbf{F}$ & M & $\mathbf{F}$ \\
\hline Toma de decisiones & 3.9 & 4.0 & 3.9 & 3.9 & 3.9 & 4.0 & 4.0 & 3.9 \\
\hline Relaciones Interpers. & 3.4 & 3.7 & 3.3 & 3.8 & 3.3 & 3.7 & 3.4 & 3.8 \\
\hline Comunic. Asertiva & 3.4 & 3.5 & 3.5 & 3.7 & 3.4 & 3.4 & 3.5 & $3.7^{*}$ \\
\hline Autoestima & 3.6 & 3.7 & $4.0\left(^{*}\right)$ & $4.1\left(^{*}\right)$ & 3.6 & 3.6 & $4.01^{*}$ & $4.1\left(^{*}\right)$ \\
\hline Manejo del estrés & 2.8 & 2.8 & $3.7\left(^{*}\right)$ & $3.8\left(^{*}\right)$ & 2.9 & 2.8 & $3.7\left(^{*}\right)$ & $3.8\left(^{*}\right)$ \\
\hline Presión grupal & 2.5 & 2.4 & $\left.4.0^{*}\right)$ & $4.3\left({ }^{*}\right)$ & 2.5 & 2.4 & $4.1\left(^{*}\right)$ & $4.2\left(^{*}\right)$ \\
\hline
\end{tabular}

$\left.{ }^{*}\right)$ Diferencia estadísticamente significativa entre el pretest y el postest, por sexo

\section{Consumo de drogas}

La prevalencia de vida del consumo de drogas se presenta en la Tabla 5. Los datos revelan que, tanto en el grupo control como aquel en que aplicó el pro- grama, se dieron aumentos significativos en la experimentación con drogas lícitas. En ambos grupos, un porcentaje importante de jóvenes que no habían consumido cuando se recogieron los datos del pretest lo hizo un año después; esta es una situación esperada 
en virtud de las edades de los jóvenes estudiados. En el caso del consumo de marihuana y cocaína, si bien los valores pre y post son diferentes, la discrepancia no es significativa, de donde se afirma que el nivel de prevalencia para ambas drogas se mantuvo igual.

El consumo activo de drogas, el cual se muestra en el Tabla 6, se mantuvo estable entre los dos períodos estudiados. Esto implica que en un año plazo, las variaciones en el consumo del último mes no fueron estadísticamente significativas, salvo en el caso del alcohol en el grupo experimental.

El consumo de drogas, tanto en su etapa experimental (consumo de vida) así como el consumo activo (prevalencia de mes) de alcohol, tabaco y marihuana por sexo, en los grupos experimental y control, se muestra en la Tabla 7. Las variaciones más fuertes se observan en el consumo activo femenino de alcohol, tabaco y marihuana, con diferencias estadísticamente significativas $(p<0,05)$ entre el pre y el postest de el grupo experimental, hallazgo que guarda coherencia con los resultados no esperados comentados en párrafos anteriores.

Al relacionarse estos datos de consumo en la dimensión postest con los puntajes obtenidos en todos los dominios, se obtiene lo expuesto en la siguiente Tabla 8. Recuérdese que el puntaje global, es una ponderación de los puntajes obtenidos en todos los dominios.

Tabla 5. Prevalencia de vida de consumo de drogas en los estudiantes de los grupos control y experimental. Costa Rica, 2001-2002. (Valores porcentuales)

\begin{tabular}{|lcccc|}
\hline DROGAS & $\begin{array}{c}\text { pretest } \\
\text { CONTROLES }\end{array}$ & $\begin{array}{c}\text { postest } \\
\text { CONTROLES }\end{array}$ & $\begin{array}{c}\text { pretest } \\
\text { EXPERIM. }\end{array}$ & $\begin{array}{c}\text { postest } \\
\text { EXPERIM. }\end{array}$ \\
\hline Alcohol & 37.4 & $45.0^{*}$ & 33.5 & $41.1^{*}$ \\
Tabaco & 11.8 & $21.0^{*}$ & 13.0 & $23.0^{*}$ \\
Marihuana & 1.0 & 1.9 & 1.0 & 2.5 \\
Cocaína & 0.3 & 0.6 & 0.4 & 0.5 \\
\hline (*) Diferencia estadisticamente significativa entre el pretest y el postest. \\
\hline
\end{tabular}

Tabla 6. Consumo activo de drogas en los estudiantes de los grupos control y experimental. Costa Rica, 2001-2002. (Valores porcentuales)

\begin{tabular}{|lcccc|}
\hline DROGAS & $\begin{array}{c}\text { pretest } \\
\text { CONTROLES }\end{array}$ & $\begin{array}{c}\text { postest } \\
\text { CONTROLES }\end{array}$ & $\begin{array}{c}\text { pretest } \\
\text { EXPERIM. }\end{array}$ & $\begin{array}{c}\text { postest } \\
\text { EXPERIM. }\end{array}$ \\
\hline Alcohol & 17.0 & 20.0 & 16.1 & $21.3^{*}$ \\
Tabaco & 7.5 & 7.1 & 7.0 & 9.8 \\
Marihuana & 0.6 & 1.7 & 0.5 & 1.0 \\
\hline (*) Diferencia estadísticamente significativa entre el pretest y el postest. & & \\
\hline
\end{tabular}

Tabla 7. Consumo de drogas por sexo según prevalencia en los estudiantes de los grupos control y experimental. Costa Rica, 2001-2002. (Valores porcentuales)

\begin{tabular}{|c|c|c|c|c|c|c|c|c|}
\hline \multirow[t]{3}{*}{ DROGA } & \multicolumn{4}{|c|}{ CONTROLES } & \multicolumn{4}{|c|}{ EXPERIMENTALES } \\
\hline & \multicolumn{2}{|c|}{ pretest } & \multicolumn{2}{|c|}{ postest } & \multicolumn{2}{|c|}{ pretests } & \multicolumn{2}{|c|}{ postest } \\
\hline & $\mathbf{M}$ & $\mathbf{F}$ & $\mathbf{M}$ & $\mathbf{F}$ & $\mathbf{M}$ & $\mathbf{F}$ & M & $\mathbf{F}$ \\
\hline \multicolumn{9}{|l|}{ Prevalencia de vida } \\
\hline Alcohol & 49.2 & 53.2 & 50.4 & 60.2 & 52.9 & 49.2 & 49.7 & 56.7 \\
\hline Tabaco & 27.2 & 19.3 & 30.9 & 24.4 & 34.4 & 20.3 & 30.1 & $29.8^{*}$ \\
\hline Marihuana & 2.0 & 0.9 & 3.4 & 1.8 & 2.7 & 1.0 & 2.7 & 3.4 \\
\hline \multicolumn{9}{|l|}{ Prevalencia de mes } \\
\hline Alcohol & 15.3 & 18.7 & 17.4 & 21.9 & 18.6 & 13.8 & 19.7 & $22.1^{*}$ \\
\hline Tabaco & 10.0 & 5.4 & 8.1 & 6.5 & 11.3 & 2.6 & 10.9 & $9.1^{*}$ \\
\hline Marihuana & 0.7 & 0.6 & 2.1 & 1.4 & 0.7 & 0.0 & 1.1 & $1.0^{*}$ \\
\hline
\end{tabular}


Pese a que los resultados expuestos en la Tabla 8, no muestran mínimos óptimos (4 puntos o más, en promedio), se encuentran algunas diferencias significativas en relación con el tabaco, en el sentido de que ser una persona abstemia arroja puntajes superiores en los dominios que ser un fumador activo $(p<0,05)$. Esto sugiere que, tanto en uno como en otro grupo, la condición de no fumador estaría relacionada con mejores puntajes en las escalas del instrumento; lo cual, a su vez, estaría evidenciando mejores habilidades en la toma de decisiones, la autoestima, las relaciones interpersonales, la comunicación asertiva, y el manejo del estrés y de la presión grupal. Además, la probabilidad de ser un consumidor activo de alcohol y marihuana es mayor si el estudiante ha fumado alguna vez en su vida. La figura siguiente ilustra esta situación. Al tomarse como referencia el postest, cabe indicar que la situación en el grupo control es prácticamente igual que en el grupo experimental, al que hace referencia la Figura 1.

En lo que al consumo de alcohol respecta, ser un abstemio o un bebedor (activo o no activo), no establece diferencias significativas en el puntaje global obtenido en los diferentes dominios estudiados. La diferencia que se encuentra en relación con el tabaco, antes comentada, podría ser el resultado de que hacia esta última droga se han dirigido claras acciones en materia de prevención y legislación que podrían explicar las diferencias anotadas.

Acerca del consumo de marihuana, un muy limitado número de casos no permite el análisis estadístico de las diferencias encontradas. No obstante, existe una tendencia que revela puntajes más elevados, es decir más favorables en términos de habilidades para vivir, en las personas abstemias.
Tabla 8. Consumo activo de drogas y abstención en los estudiantes de los grupos control y experimental, según puntaje global en todos los dominios. Costa Rica, 2001-2002

\begin{tabular}{lcc|}
\hline TIPO DE & CONTROL \\
CONSUMO & Postest & $\begin{array}{c}\text { EXPERIMENTAL } \\
\text { Postest }\end{array}$ \\
\hline
\end{tabular}

$\begin{array}{lll}\text { Alcohol } & & \\ \text { Sí activo } & & \\ \text { No activo } & 3.86 & 3.90 \\ \text { Abstemio } & 3.90 & 3.88 \\ & 3.89 & 3.93\end{array}$

\section{Tabaco}

$\begin{array}{lcc}\text { Sí activo } & 3.75^{*} & 3.76^{* *} \\ \text { No activo } & 3.85 & 3.86 \\ \text { Abstemio } & 3.91 & 3.94\end{array}$

\section{Marihuana}

$\begin{array}{lll}\text { Sí activo (+) } & 3.44 & 3.74 \\ \text { No activo } & 3.99 & 3.91 \\ \text { Abstemio } & 3.90 & 3.91\end{array}$

* Diferencia estadísticamente significativa entre consumidores activos y abstemios

en el grupo control.

* Diferencia estadísticamente significativa entre consumidores activos y abstemios

en el grupo experimental

+ Tamaño de muestra insuficiente para consumo activo de marihuana

- Consumió en los últimos treinta días

- No consumió en los últimos treinta días, pero sí hace más tiempo

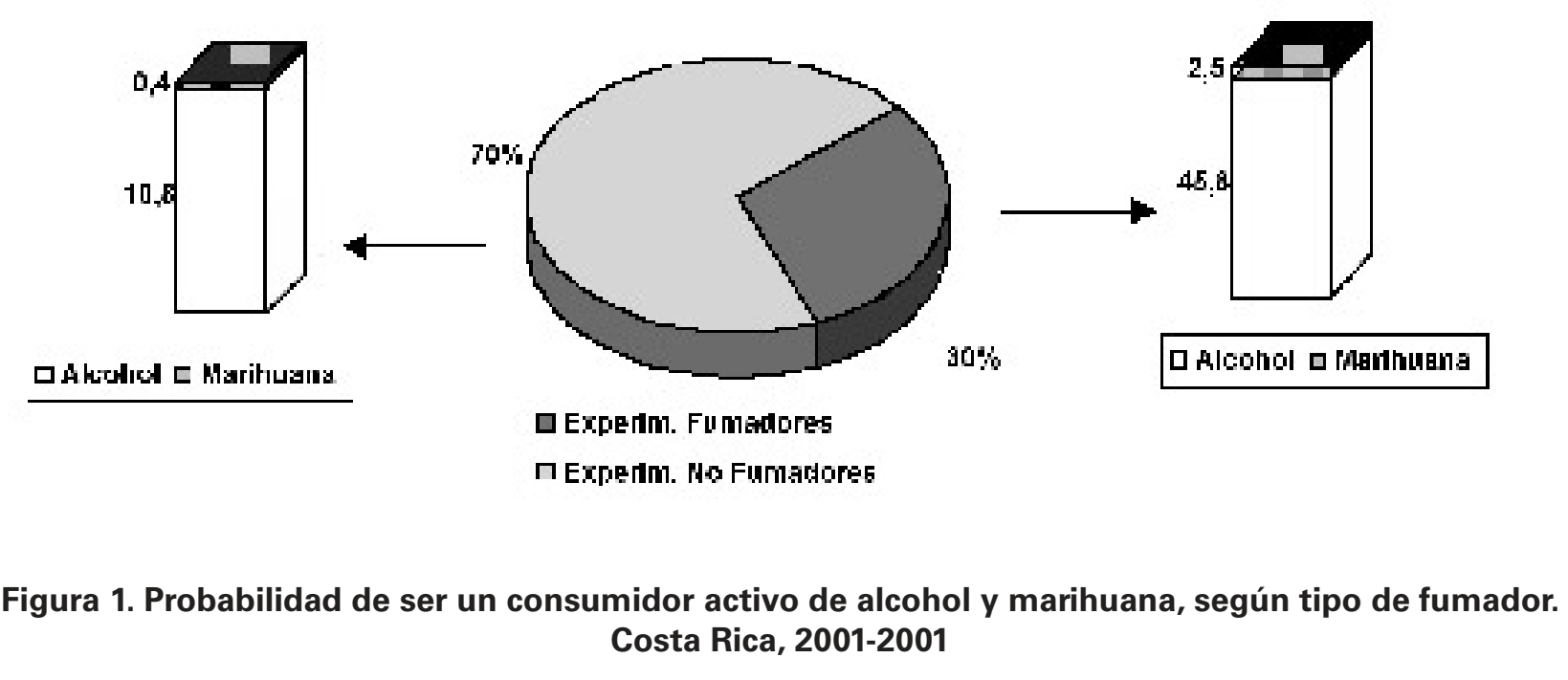


Conocimientos y actitudes hacia el tabaco y el alcohol

Las únicas variaciones significativas observadas tanto en los controles como en el grupo experimental, tuvieron lugar en el área de los conocimientos. En el caso de los conocimientos hacia el consumo de alcohol y el tabaco, el grupo experimental muestra un aumento significativo de los conocimientos $(p<0,05)$. Si bien no se registraron diferencias significativas en la sección de actitudes cabe destacar que los puntajes obtenidos son adecuados, al ser mayores de 4 puntos.

Al contrastar el tipo de actitud y conocimientos hacia el alcohol y el tabaco con el desempeño en todos los dominios, se obtienen los resultados expuestos en la Tabla 10, los cuales indican que los individuos que tienen un buen desempeño en los dominios tienen a su vez altos puntajes en actitudes hacia el tabaco (actitudes de rechazo hacia el fumado). Esta misma relación se encuentra en el grupo en el cual se aplicó el programa, cuando se analiza su actitud hacia las bebidas alcohólicas y hacia el tabaco, encontrándose que los estudiantes con mejor desempeño en los dominios, tienen una actitud de mayor rechazo hacia la conducta de beber y fumar, pese a que el puntaje exhibido en alcohol no es el óptimo.

\section{DISCUSIÓN}

Varios cuestionamientos surgen a partir de esta evaluación, mismos que también han estado presentes en otras realizadas fuera del ámbito nacional.

Tabla 9. Puntajes $(* *)$ obtenidos por los estudiantes en el pre y el postest en las escalas de conocimientos y actitudes hacia el alcohol y el tabaco. Costa Rica, 2001-2002. (Valores porcentuales)

\begin{tabular}{|c|c|c|c|c|}
\hline \multirow[t]{2}{*}{ Actitudes/Conocimientos } & \multicolumn{2}{|c|}{ CONTROLES } & \multicolumn{2}{|c|}{ EXPERIMENTALES } \\
\hline & pretest & postest & pretests & postest \\
\hline \multicolumn{5}{|l|}{ Actitudes } \\
\hline Alcohol & 4,23 & $4,09 *$ & 4,12 & 4,05 \\
\hline Tabaco & 4,06 & 4,06 & 4,02 & 3,95 \\
\hline \multicolumn{5}{|l|}{ Conocimientos } \\
\hline Alcohol & 3,42 & 3,50 & 3,37 & $3,53 *$ \\
\hline Tabaco & 4,24 & $4,41 *$ & 4,20 & $4,34 *$ \\
\hline
\end{tabular}

Tabla 10. Puntajes $\left(^{* *}\right)$ obtenidos por los estudiantes en el postest, según desempeño en todos los dominios. Costa Rica, 2001-2002. (Valores porcentuales)

\begin{tabular}{|c|c|c|c|c|}
\hline \multirow[t]{3}{*}{ Actitudes/Conocimientos } & \multicolumn{2}{|c|}{ CONTROL } & \multicolumn{2}{|c|}{ EXPERIMENTAL } \\
\hline & \multicolumn{2}{|c|}{ Desempeño en dominios $^{\circ}$} & \multicolumn{2}{|c|}{ Desempeño en dominios ${ }^{\circ}$} \\
\hline & Inadecuado & Adecuado & Inadecuado & Adecuado \\
\hline \multicolumn{5}{|l|}{ Actitudes } \\
\hline Alcohol & 3,50 & 3,50 & 3,48 & $3,58 *$ \\
\hline Tabaco & 3,98 & $4,18 *$ & 3,84 & $4,10 *$ \\
\hline \multicolumn{5}{|l|}{ Conocimientos } \\
\hline Alcohol & 3,50 & 3,50 & 3,48 & $3,58 *$ \\
\hline Tabaco & 4,39 & 4,45 & 4,27 & 4,45 \\
\hline
\end{tabular}


En primer lugar, surge la pregunta de si un año plazo es tiempo suficiente para estimar de manera confiable los efectos producidos por un programa de prevención. En criterio de los autores la respuesta amerita ser relativizada pues el nivel de conocimiento actual en el país sobre el efecto de los programas de prevención es aún incipiente. Probablemente quince sesiones de trabajo distribuidas en un año pudieran ser insuficientes para modificar actitudes y prácticas vinculadas con el consumo de drogas, las cuales necesariamente han sido parte de la vida de los jóvenes desde temprana edad; es decir, la exposición a la publicidad del tabaco y a la del alcohol, aunadas a actitudes con diferentes grados de permisividad de los adultos, son fenómenos que están presentes desde la niñez y configuran estilos y formas de reaccionar particulares. Esto podría sugerir la importancia no sólo de desarrollar el trabajo preventivo, y por ende evaluativo, dentro de plazos más extensos, sino iniciarlo a edades más tempranas, situación que en Costa Rica se empieza a trabajar actualmente desde el 4to grado de la enseñanza primaria. Asimismo, los esfuerzos preventivos deberían igualmente abarcar el nivel internacional, la presión ejercida por medios como la Internet, la televisión por cable y el cine, entre otros, deberían incluir la promoción de estilos saludables de vida, en la búsqueda de un mundo mejor. En lo que atañe al programa preventivo como tal, cabe anotar como principal limitación la ausencia de una evaluación cualitativa que permitiera establecer tanto con los docentes como con los estudiantes las características y la calidad en su aplicación.

Evaluar un programa a un año plazo, en personas que se encuentran en edades en las que normalmente suele iniciarse el proceso de experimentación de conductas y la búsqueda de nuevas vivencias, pone de relieve a su vez un cuestionamiento central en torno a la idea de la extensión de los programas y los períodos de evaluación.

Por otra parte, existe evidencia internacional de que los efectos de un programa preventivo pudieran observarse a corto plazo y que, contrariamente a la idea de que el paso del tiempo consolidaría los cambios en el comportamiento, más bien este provocaría un declive en los mismos. Estas son consideraciones que deben someterse a prueba en el medio costarricense, toda vez que este tipo de experiencias son completamente novedosas.

Es importante recalcar que esta evaluación de un programa preventivo queda limitada a su estudio dentro de cortos plazos, debido principalmente a los pocos recursos financieros y humanos destinados a la investigación - evaluación.

Entre los aspectos que llaman la atención en este estudio, sobresale la mejoría que tanto el grupo de intervención como el grupo control mostraron en varios de los dominios. La autoestima, el manejo del estrés, de la presión grupal y la comunicación asertiva son áreas que mejoraron en unos y en otros. Posiblemente este logro deba adjudicarse al programa, pero también es probable que los jóvenes, tanto en uno como en otro grupo, estuviesen expuestos a influencias preventivas que escaparon del control y manejo de los investigadores. Un ejemplo de esta situación sería la prevención del tabaquismo, difundida por los medios de comunicación en el nivel nacional y, probablemente, el desarrollo de otras acciones de signo semejante impulsadas por los colegios, el estado, otras organizaciones y ciertas iniciativas privadas. En este sentido, el efecto de la acción preventiva no se puede considerar inocua o, al menos debería ser objeto de nuevas exploraciones.

La impresión general que subyace a los resultados de este estudio es que existe, luego de un año de desarrollo del programa, una serie de cambios que inicialmente pudieran ser relativamente modestos pero que, quizás, su evaluación en un plazo mayor, a la par de programas continuos de prevención, pudiera mostrar resultados más coherentes con lo que se esperaría de acuerdo con los objetivos.

Al considerarse los resultados en términos del consumo de drogas, cabe anotar una aparente paradoja en los mismos, dado que la experimentación aumentó, así como el consumo activo de los grupos en los que se aplicó el programa y en los cuales se esperarían aumentos leves o, al menos, estabilidad. Estos resultados, no obstante, se asemejan a los obtenidos por Corvo y Persse (1998) en poblaciones de preecolares al evaluar las expectativas de los niños en relación con el alcohol. De igual manera, se podrían citar los estudios de Kraus, Smith y Ratner (1994) quienes inexplicablemente encontraron un aumento en las expectativas hacia el alcohol luego de un programa tendiente a reducirlas. Debe considerarse que los jóvenes evolucionan, sobre todo en las edades estudiadas y, aparentemente, el programa no habría podido influir de manera medible y significativa sobre esta evolución.

Las diferencias observadas entre hombres y mujeres, en el sentido de un mejor desempeño femenino en los dominios, entre el pre y el postest, podría constituir un primer elemento para que se considere la implementación de estrategias de prevención diferenciadas según el género.

Un hallazgo relevante de esta investigación se refiere a la importancia preventiva de la condición de ser un abstemio en el consumo de tabaco y presentar una estado favorable en los dominios evaluados, en comparación con los estudiantes que fuman activamente. Vinculado con lo anterior destaca el hecho que los estudiantes con un buen nivel en las habilidades para vivir presentan, a su vez, mejores conocimientos sobre el alcohol y el tabaco (especialmente en el grupo experimental) y actitudes de mayor recha- 
zo hacia el tabaco y el alcohol (también en el grupo experimental), en comparación con aquellos cuyos puntajes en los dominios no fue adecuado. De lo anterior se puede deducir que la existencia de niveles apropiados en las habilidades para vivir, se asocian con actitudes de rechazo hacia el tabaco lo cual, a su vez, estaría revelando la importancia de que los niños y los jóvenes desarrollen buenas habilidades para la vida, desde muy temprano, para facilitar actitudes de rechazo hacia el tabaquismo y, consecuentemente hacia el consumo de otras sustancias psicoactivas, pues como se ha observado en este estudio la probabilidad de consumir drogas ilícitas (marihuana y cocaína) es considerablemente menor si el joven no fuma.

\section{REFERENCIAS}

Becoña, E. (1999). Bases teóricas que sustentan los programas de prevención de drogas, Ministerio de Interior, Madrid

Bejarano, J., Carvajal, H. y San Lee, L. (1996). Consumo de Drogas en Costa Rica. Resultados de la encuesta nacional de 1995. San José, C.R.: IAFA.

Bejarano, Julio (1999). El consumo de tabaco en la población joven costarricense. Hallazgos de la encuesta mundial sobre tabaco en jóvenes (GYTS). Revista Costarricense de Salud Pública, Año 10, 18 y 19 (19-25).

Bejarano, Julio y San Lee, Lizú. (1997). El fenómeno Social de la droga en la juventud costarricense, Revista Costarricense de Psicología, 28, (9-24).

Botvin, G., Baker, E. et.al. (1990). Preventing adolescent drug abuse through a multimodal cognitive-behavioral approach: Results of a three-year study. Journal of Counsulting and Clinical Psychology, 58, (437-446).

Botvin, G., et.al. (1995). Long-term follow up results of a randomized drug-abuse prevention trial in a white class population, Journal of the American Medical Association, 273, (1106-1112).

Corvo, K. \& Persee, L. (1998). An evaluation of a preeschool based prevention program: longitudinal effects on children's alcohol-related expectancies. Journal of Alcohol and Drug Education, 43, 2, (36-47).

Elso, J. et.al. (2000) Drogas y escuela VI. Bilbao, ES: Gráficas Lankorpe, S.L.

Espada, J.P, Méndez, F., e Hidalgo, M. (2000). Consumo de alcohol en escolares: descenso de la edad de inicio y cambios en los patrones de ingesta. Revista Adicciones Vol. 12, 1 (57-64).
Gorman, D.M. (1996). Ethiological theories and the primary prevention of drug use. Journal of Drug Issues, 26, (505-520).

ICD. (2003). Plan Nacional Anti-Drogas 2005 - 2007. Instituto Costarricense sobre Drogas: San José, Costa Rica.

Instituto sobre Alcoholismo y Farmacodependencia (2000) Habilidades para vivir. Trazando el Camino. San José, C.R.: IAFA

Instituto sobre Alcoholismo y Farmacodependencia (2001) Trazando el camino, 9. Año. San José, C.R.: Imprenta Nacional.

Joya, R. Habilidades para vivir. Trazando el Camino, Sétimo año (1999). San José, C.R.: Imprenta Nacional.

Krausskopf, Dina (1994). Adolescencia y Educación Segunda Edición. San José, C.R.: Editorial Universidad Estatal a Distancia,

Likert, R. (1976). Una técnica para la medición de actitudes, escalas de medición en ciencias sociales. Buenos Aires: Ediciones Nueva Visión.

Mideplan. (2001). Plan Nacional de Desarrollo 2002 - 2006. San José, Costa Rica.

Ministerio de Salud. (2001). Plan Nacional de Salud 2002 - 2006. San José, Costa Rica.

Obando, P. y Sáenz, M. (2001) El fenómeno droga en la población escolar de la provincia de San José. Tibás, 2000. Revista de Ciencias Sociales, 94, (35-49).

OMS (1993). Enseñanza en los colegios de las habilidades para vivir. Ginebra, OMS, División de Salud Mental.

OPS/OMS, I.I.P./ U.C.R (1999) Conducta de Riesgo en adolescentes de 12 a 19 años en Costa Rica. Primera edición, San José, C.R. Ediciones Perro Azul.

Padua, J. (1979) Técnicas de investigación aplicadas a las ciencias sociales. México: El Colegio de México, Fondo de la Cultura Económica.

Papalia, D. y Olds, S. (1990). Psicología del desarrollo: de la infancia a la adolescencia, México: McGraw Hill.

Proyecto Estado de la Nación (2001).Estado de la Nación en Desarrollo Humano Sostenible. Séptimo Informe 2000. Primera edición, San José, C.R.

Scheier, L.; et.al. (1999) Social skills, competence, and drug refusal efficacy as predictors of adolescent alcohol use. Journal of Drug Education, 29 3, 251-278.

Ugalde, Federico, et.al. (2003). Análisis del consumo de drogas en adolescentes escolarizados de Costa Rica, 1999. Revista de Ciencias Sociales, 99, (11-23).

Valverde, Oscar, et.al. (2001). Adolescencia, protección y riesgo en Costa Rica. Encuesta Nacional sobre conductas de Riesgo en los y las Adolescentes de Costa Rica. San José: CCSS, Programa Atención Integral a la Adolescencia. 